\title{
ORIGINAL ARTICLE \\ Feasibility of an online well-being intervention for people with spinal cord injury: a pilot study
}

\author{
JH Verwer ${ }^{1}$, CMC van Leeuwen ${ }^{1,2}, \mathrm{~L}_{\text {Bolier }}^{3}$ and MWM Post ${ }^{1,2,4}$
}

Study design: Pre-test and post-test designs with 14 participants. Measurements were taken at baseline (T1), immediately after the intervention (T2) and at 3-month follow-up (T3).

Objectives: Psyfit is an online self-help program designed to enhance well-being in persons with depressed mood. We examined the feasibility of Psyfit in people with spinal cord injury (SCI).

Setting: Community, the Netherlands.

Methods: Participants chose two of the six Psyfit modules. The researcher maintained telephone contact with the participants. Feasibility was inferred from the completion rate of the modules and feedback from the participants. Outcome measures were the Mental Health Inventory-5, the Center for Epidemiological Studies Depression scale and the Warwick-Edinburgh Mental Well-Being Scale.

Results: Overall, $75 \%$ of the first module and $39 \%$ of the second module were completed. Seven participants were considered as study completers and were included in the evaluation. They evaluated Psyfit as a useful program and helpful for persons with SCl. Several technical problems were reported that mainly concerned browser compatibility. An increase in mental health and nonsignificant change of well-being were found at the end of the intervention period, but these were not maintained at follow-up.

Conclusion: Psyfit seems a potentially feasible program. However, adaptation to the SCl population and further study with a controlled design and utilizing a larger sample size are necessary before it can be recommended as part of SCl rehabilitation.

Spinal Cord (2016) 54, 473-477; doi:10.1038/sc.2015.165; published online 20 October 2015

\section{INTRODUCTION}

Many people with spinal cord injury (SCI) experience poor mental health and well-being. ${ }^{1}$ Specialized psychological care for people with SCI is, however, sparse, in particular in the outpatient situation. In such situations, the Stepped-Care model provides a useful perspective. ${ }^{2,3}$ In this model, timing and intensity of care are tailored to the needs of the person involved. With aggravating depressive symptoms, more thorough interventions, like using medication and psychotherapy, are used. ${ }^{2}$ In case of mild symptoms, however, inperson treatment may not be optimal. Availability issues (for example, waiting lists), stigma in society about psychological therapy and norms of self-reliance and self-sufficiency might prevent patients from searching for treatment. ${ }^{4}$ Internet-based psychological treatment might be an accessible, effective and low-cost intervention in people with mild mental health problems. ${ }^{5}$ There is, however, hardly any evidence for the application of such interventions in people with SCI. ${ }^{6}$ We could only find one small study in which an online psychological program resulted in reduction in symptoms of depression, anxiety or stress in all three participants who had completed the program. ${ }^{7}$ The 'ePACT' program evaluated in that study is a psychological therapy based on cognitive behaviour and positive psychology. It contains 10 sessions and 4 information pages and addresses problem-solving, altered thinking, goal setting and relaxation, among others.
Psyfit is an online self-help intervention that has shown effectiveness in people with mild to moderate depressive symptoms in the Netherlands. ${ }^{8}$ Psyfit is based on positive psychology, mindfulness, cognitive behavioural therapy and problem-solving therapy and aims to strengthen personal resources and qualities in order to, primarily, enhance mental well-being and, secondarily, reduce symptoms of depression and anxiety. ${ }^{8}$ It is available at a very low cost of about $€ 30$ per year, and several health insurance companies have made it available for free for their clients. If applicable in people with SCI, Psyfit would therefore be an attractive option for people with SCI who experience mild depressive symptoms before they should consider more intensive treatment modalities.

This pilot study was designed to examine the feasibility of Psyfit for use in people with SCI. Second, we investigated whether the application of Psyfit might enhance mental health and well-being and reduce depressive symptoms in this population.

\section{MATERIALS AND METHODS}

Design

This pilot study used a pre-test/post-test design. Measurements were taken at baseline (T1), immediately after the intervention (T2) and at 3-month follow-up (T3).

\footnotetext{
${ }^{1}$ Center of Excellence in Rehabilitation Medicine, De Hoogstraat Rehabilitation, Utrecht, The Netherlands; ${ }^{2}$ Brain Center Rudolf Magnus and Center of Excellence in Rehabilitation Medicine, Rudolf Magnus Institute of Neuroscience, De Hoogstraat Rehabilitation, University Medical Center Utrecht, Utrecht, The Netherlands; ${ }^{3}$ Department of Public Mental Health, Trimbos Institute, Utrecht, The Netherlands and ${ }^{4}$ Department of Rehabilitation Medicine, Center for Rehabilitation, University of Groningen, University Medical Center Groningen, Groningen, The Netherlands

Correspondence: Dr CMC van Leeuwen, Brain Center Rudolf Magnus and Center of Excellence for Rehabilitation Medicine, Rudolf Magnus Institute of Neuroscience, De Hoogstraat Rehabilitation, University Medical Center Utrecht, Rembrandtkade 10, Utrecht 3585TM, The Netherlands.

E-mail: c.v.leeuwen@dehoogstraat.nl

Received 27 October 2014; revised 30 August 2015; accepted 4 September 2015; published online 20 October 2015
} 


\section{Participants}

The participants were recruited through two Dutch rehabilitation centres specializing in SCI rehabilitation, De Hoogstraat in Utrecht and Sint Maartenskliniek in Nijmegen. Eligibility was assessed by the rehabilitation psychologist of the centre. Inclusion criteria were SCI, having finished outpatient rehabilitation, being in a mental recovery process and known to have mild to moderate symptoms of depressed mood to the psychologist. Exclusion criteria were SCI due to malignity, insufficient understanding of the Dutch language, insufficient computer affinity and insufficient hand or chin function to use a computer.

For practical reasons, we only involved participants in the current evaluation, who completed at least one module, had started with a second module and performed the post-test $\mathrm{T} 2$.

\section{Intervention}

Psyfit consists of six independent modules: (1) positive feelings, (2) positive relations, (3) mindfulness, (4) optimistic thinking, (5) mastering your life and (6) personal mission statement and goal setting. ${ }^{8}$ Each module is to be completed in 4 weeks. In each week, a specific topic is addressed, including general information, psycho-education and a practical exercise. All exercises have to be completed before one can start with the next weeks' content and one can start with the next step of the program on first day of next week. With a self-test at the start and the end of each module, the participant can monitor his own improvement. In the original study, ${ }^{8}$ participants were advised to do at least 1 module. Each module is an intervention on its own and can have benefits. Participants were also free to choose the order and the number of modules to complete. The results showed that participants started on average 1.39 modules. In this pilot study, we therefore asked participants to choose and complete two of the six modules.

\section{Procedure}

Participants were recruited by the two psychologists in the participating rehabilitation centres. Each participant was informed about the study and invited for an intake with the psychologist and the researcher. In this intake, Psyfit was introduced, and information about the study was provided. After informed consent was obtained, the participant completed the pre-test questionnaires. Then, all six modules of Psyfit were introduced to the participant, who then explained his/her personal reasons of poor mental health. The psychologist and researcher explained the module(s) that would best suit to that situation, and, after deliberation with the participant, the two modules were chosen.

During the intervention, the researcher stayed in contact with each participant by phone every 2 weeks to inquire about their progress, experiences and questions and to encourage them to continue with the program. The researcher performed the post-test in person, whereas the self-administered standardized questionnaires on the 3-month follow-up were sent via mail or e-mail.

\section{Instruments}

Feasibility of Psyfit. Adherence: the completion rate of each module and the proportion of participants completing one and two modules were computed. A module was considered completed if the participant completed at least 50\% of the module. A participant who had completed $100 \%$ of the first module and had made a start with the second module was considered a study completer. A participant who did not start a second module was considered a dropout.

Satisfaction: examples of questions were as follows: 'which exercise did you like the best and which the least' and 'what would you change about Psyfit?'. The interview took on average $60 \mathrm{~min}$. The proportion of study completers evaluating Psyfit positively, and those who would recommend Psyfit to other persons with SCI, was computed. Positive and negative feedback on Psyfit was collected, and comments given by two or more participants were reported.

Mental health, depression and well-being. The Mental Health Index-5 (MHI-5) was used to assess mood during the last 4 weeks, with higher scores representing better mental health. The MHI- 5 consists of five items and was found reliable and valid in the SCI population. ${ }^{9}$
The Center for Epidemiological Studies Developmental scale (CES-D) measures symptoms of depression during the last week, with high scores representing more severe symptoms of depression. It consists of 20 items and was found reliable and valid in the SCI population. ${ }^{10}$

The Warwick-Edinburgh Mental Well-Being Scale (WEMWBS) assesses mental well-being in the last 2 weeks, with high scores representing good mental well-being. The scale consists of 14 items and was shown to be valid. ${ }^{11}$

\section{Data analysis}

The interviews were recorded and transcribed. Researchers (JV and CvL) independently coded the data. Codes were compared and discussed until consensus was reached. The qualitative data were split into the following: general and personal feedback; content-related feedback (text and exercises); feedback concerning the form of the program (length, frequency); technical errors; and feedback with regard to the support by the researcher.

Differences in the MHI-5, CES-D and WEMWBS scores between the three time points were tested using the Wilcoxon Signed-Rank test. Effect sizes were calculated with the formula:

$$
\mathrm{R}=\mathrm{Z} / \sqrt{ }(\mathrm{N})
$$

\section{Statement of ethics}

We certify that all applicable institutional and governmental regulations concerning the ethical use of human volunteers were followed during the course of this research. The Medical Ethics Committee of the University Medical Centre Utrecht declared that this protocol did not need formal ethical approval under the Dutch law regulating medical research in human beings. The local medical committees of both participating rehabilitation centres approved this trial.

\section{RESULTS}

\section{Respondent characteristics}

In total, 22 persons (11 in each rehabilitation centre) were invited for the study. Eight declined participation, leading to a total of 14 participants in the study (Table 1).

Adherence. Table 2 shows the two chosen modules per participant, along with the completion rate and the time to completion, if applicable. On average, the 14 participants completed $75 \%$ of the first module and $39 \%$ of the second module. Of the 14 participants, 11 participants completed $50 \%$ or more of the first module. Seven completed $100 \%$ of the first module and started with the second module and were considered study completers. Five of them completed at least $50 \%$ of the second module, and two participants fully completed both modules. All modules were chosen by one or more participants. Most dropouts started with the module 'mastering your life' (4).

Table 1 shows that study completers were younger, had a higher level of education and had more often an incomplete lesion compared with dropouts.

For three dropouts Psyfit presented them with psychological challenges and overwhelming emotions associated with the rehabilitation period. For one dropout, Psyfit was not what she expected, two reported to have other priorities at the time and one could not be contacted any more after the first telephone contact. The dropouts did not perform the post test or the 3-month follow-up.

Satisfaction. Five of the seven study completers evaluated Psyfit as overall a good program, and five would recommend Psyfit to another person with SCI. Eight technical problems were reported, of which seven concerned browser compatibility and came from two persons 
using an iPad. Another participant experienced problems signing up with a Polish e-mail address.

Table 3 presents the main qualitative feedback of the study completers. Feedback was sometimes conflicting, for example, the desired amount of reminders and accessibility through e-mail, and none of the study completers were unambiguously positive or negative. Their main criticism concerned the generic nature of Psyfit, that is, not being adapted for use in people with SCI. Six study

Table 1 Characteristics of the Psyfit participants and differences between study completers and dropouts

\begin{tabular}{|c|c|c|c|}
\hline Variable & $A / l(N=14)$ & $\begin{array}{l}\text { Completers } \\
\qquad(N=7)\end{array}$ & $\begin{array}{l}\text { Dropouts } \\
(N=7)\end{array}$ \\
\hline $\begin{array}{l}\text { Age (mean, min-max } \\
\text { in years) }\end{array}$ & $44.7(21-63)$ & $41.7(26-63)$ & $47.9(21-63)$ \\
\hline Gender (male:female) & $11: 3$ & $6: 1$ & $5: 2$ \\
\hline $\begin{array}{l}\text { Living area (region Utrecht: } \\
\text { Nijmegen) }\end{array}$ & $7: 7$ & $4: 3$ & $3: 4$ \\
\hline \multicolumn{4}{|l|}{ Education $(N)^{a}$} \\
\hline Low & 2 & 0 & 2 \\
\hline Medium & 4 & 2 & 2 \\
\hline High & 8 & 5 & 3 \\
\hline \multicolumn{4}{|l|}{ Occupational status (N) } \\
\hline Paid work & 3 & 2 & 1 \\
\hline Volunteer work & 1 & 0 & 1 \\
\hline Other & 4 & 3 & 1 \\
\hline Inactive & 6 & 2 & 4 \\
\hline Marital status ( $N$ single) & 4 & 3 & 1 \\
\hline Type of SCl ( $N$ paraplegia) & 10 & 5 & 5 \\
\hline Motor completeness ( $N$ complete) & 6 & 2 & 4 \\
\hline $\begin{array}{l}\text { Months since injury } \\
\text { (median, min-max) }\end{array}$ & $15(6-36)$ & $13(6-36)$ & $18(9-29)$ \\
\hline
\end{tabular}

aLow education: no or only vocational education; medium education: high school; high education: bachelor/master. completers suggested to add references to SCI in the module or at least add short phrases as 'if possible in your personal physical situation'. Further, four completers recommended to add underlying theory to the modules to enable them to better understand the background and purpose of the exercises and to be able to adapt these to their situation. Other criticisms concerned the module length and rigidity. Suggestions were made to make the programme more compact and flexible, for example, enabling change of the day on which each week starts and ends. Finally, several completers preferred to be able to skip unsuitable exercises.

Changes in mental health, depressive symptoms and well-being. Table 4 presents the mental health and well-being scores of the participants, along with effect sizes. The dropouts showed slightly better scores at T1 than the study completers, but these differences were not significant. The completers showed a significant improvement of the MHI scores between T1 and T2. However, all scores decreased significantly between T2 and T3, and no significant change was seen between $\mathrm{T} 1$ and $\mathrm{T} 3$.

\section{DISCUSSION}

This pilot study of a generic e-health well-being intervention showed that most participants completed at least one of the modules and that the completers were generally positive about Psyfit. However, they were less positive about the suitability for persons with SCI and the flexibility of the program. A positive, but temporary, increase in mental health was found at post test.

\section{Feasibility}

The adherence of the current study was similar to the results of other online psychological interventions. ${ }^{7,8,12}$ Still, the amount of participants not completing one module was considerable in this study, despite the intensive telephone support. Different factors may influence adherence to this type of treatment, such as disease severity ${ }^{13}$ and the ability to reflect on one's thoughts and feelings that such programmes require. ${ }^{14}$

Table 2 Name and final completion rate of first and second module per participant

\begin{tabular}{|c|c|c|c|c|c|c|}
\hline \multirow[t]{2}{*}{ Participant } & \multicolumn{3}{|c|}{ First module } & \multicolumn{3}{|c|}{ Second module } \\
\hline & Title & Completion rate (\%) & Time to complete (days) & Title & Completion rate (\%) & Time to complete (days) \\
\hline 1 & Mastering your life & 64 & NA & Mindfulness & NS & NA \\
\hline 2 & Mindfulness & 32 & NA & Mastering your life & NS & NA \\
\hline 3 & Optimistic thinking & 100 & 31 & Positive relationships & 100 & 39 \\
\hline 4 & Mission and goals & 100 & 33 & Positive feelings & 89 & NA \\
\hline 5 & Positive feelings & 100 & 93 & Optimistic thinking & 47 & NA \\
\hline 6 & Positive relationships & 100 & 40 & Mindfulness & 32 & NA \\
\hline 7 & Positive feelings & 100 & 30 & Mastering your life & 88 & NA \\
\hline 8 & Mission and goals & 45 & NA & Optimistic thinking & NS & NA \\
\hline 9 & Mastering your life & 100 & 43 & Positive relationships & 90 & NA \\
\hline 10 & Mastering your life & 28 & NA & Optimistic thinking & NS & NA \\
\hline 11 & Mission and goals & 60 & NA & Positive feelings & NS & NA \\
\hline 12 & Positive feelings & 100 & 75 & Mindfulness & 100 & 47 \\
\hline 13 & Mastering your life & 52 & NA & Positive feelings & NS & NA \\
\hline 14 & Mastering your life & 68 & NA & Positive feelings & NS & NA \\
\hline
\end{tabular}

Abbreviations: NA, not applicable; NS, not started. 
Table 3 Feedback about Psyfit by study completers ${ }^{a}$

\begin{tabular}{|c|c|}
\hline \multicolumn{2}{|l|}{ Positive feedback } \\
\hline Personal/general feedback & $\begin{array}{l}\text { Sound, logical program in general (5) } \\
\text { Would recommend Psyfit to peers with } \mathrm{SCl}(5) \\
\text { At least one module was personally relevant (4) } \\
\text { Interested in other modules (3) } \\
\text { Psyfit provided at least some help in psychological adaptation to the } \mathrm{SCl} \text { (2) }\end{array}$ \\
\hline Content (text and exercises) & $\begin{array}{l}\text { Decent exercises, with examples, focusing on personal problems (5) } \\
\text { Clear videos (providing extra value) (3) }\end{array}$ \\
\hline Technical (program concept, software, logistics, web-support) & $\begin{array}{l}\text { Accessible system through period of } 4 \text { weeks/good module length (4) } \\
\text { No technical problems (4) }\end{array}$ \\
\hline Supervision by telephone & $\begin{array}{l}\text { Adequate supervision, sound examples, explanations, feeling of being stimulated ( } 7 \text { ) } \\
\text { Frequency of contact was fine (3) }\end{array}$ \\
\hline \multicolumn{2}{|l|}{ Negative feedback } \\
\hline Personal/general feedback & $\begin{array}{l}\text { No extra benefit in context of psychological process of } \mathrm{SCl} \text { (variety of reasons) (5) } \\
\text { Motivation decreased (due to variety of reasons) (5) } \\
\text { Second module less topical (2) }\end{array}$ \\
\hline Content (text and exercises) & $\begin{array}{l}\text { Needs to refer to } \mathrm{SCl} \text { in the text and exercise (add a phrase like 'try if possible, or alternatively, ...') (6) } \\
\text { Add more theory/background (4) } \\
\text { More exercises would be nice (2) }\end{array}$ \\
\hline Technical (program concept, software, logistics, web-support) & $\begin{array}{l}\text { Make modules more compact: four exercises in } 2 \text { weeks (4) } \\
\text { Make program more flexible (3), for example, by changing start of the week, shortening the week } \\
\text { Opportunity to print/export exercises (2) } \\
\text { Browser compatibility problems for iPad (videos, typing in textboxes, unable to close pop-up windows) (5) } \\
\text { Unable to get access to the new week (2) }\end{array}$ \\
\hline
\end{tabular}

aNumbers in parentheses indicate the number of participants providing this comment.

Table 4 Mental health and well-being scores at baseline and follow-up $(N=7$, median (IQR))

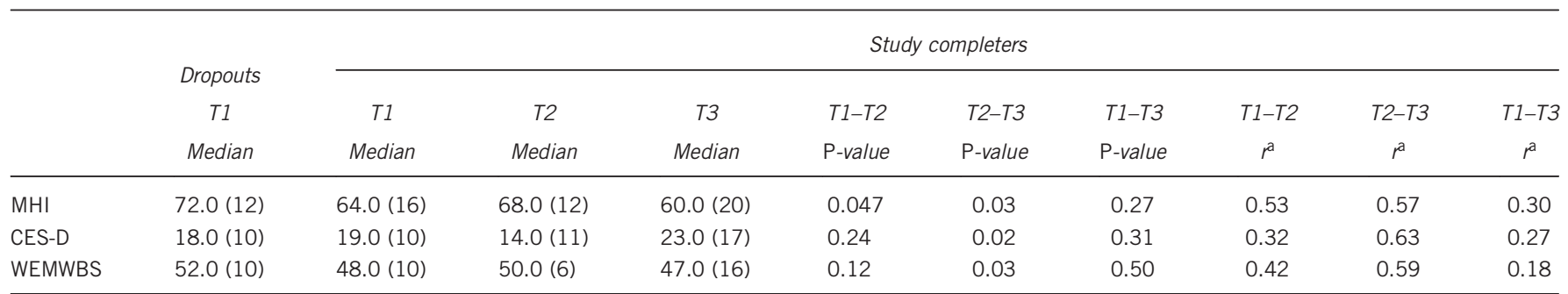

Abbreviations: CES-D, Center for Epidemiological Studies Developmental scale; IQR, interquartile range; MHI, Mental Health Index; WEMWBS, Warwick-Edinburgh Mental Well-Being Scale. ${ }^{a} r=$ effect size $0.1=$ small effect, $0.3=$ medium effect, $0.5=$ large effect.

\section{Satisfaction}

Most completers evaluated Psyfit as a useful program, indicating that this program may be of use to at least part of the SCI population. Adaptation of this program was, however, recommended to make it better suited to people with SCI. Completers also recommended making the programme more flexible. In our opinion, Psyfit is most suitable for relatively well-educated persons with the ability and willingness to reflect on one's feelings and thoughts and who are comfortable using the internet.

Changes in mental health, depressive symptoms and well-being Completing at least one of the six independent modules of Psyfit was associated with a significant, but temporary, increase in mental health. Migliorini et al. ${ }^{7}$ also found improvement of mood after completion of their intervention, and this effect was neither universal nor significant. So far, the results of online positive psychological interventions do not compare positively with the results of traditional or telephone-based cognitive behavioural therapy of depression in persons with SCI. ${ }^{15,16}$
In the original evaluation of Psyfit, Bolier et al. ${ }^{8}$ reported significant small to moderate effects in persons in the general population, and these effects were larger. It is unclear why in our study, after encouraging short-term results, the longer-term (3 months post intervention) results were disappointing. This finding might indicate that it is difficult to keep up the necessary motivation to do the exercises. A more tailored intervention that makes use of persuasive technologies, ${ }^{17}$ personalized to the needs and preferences of the participant, accompanied with personal guidance, seems to be recommended to induce longer-lasting change.

Limitations. The current study was designed as a pilot study; therefore, the sample size was very small, and firm conclusions about treatment effects cannot be drawn. Second, this study lacked a control group, and therefore it is also difficult to evaluate the true intervention effects and check for possible spontaneous change. Third, only participants who were considered a completer were included in the 
evaluation; hence, our results might suffer from bias towards more positive outcomes.

Recommendations for future research. It is recommended to replicate the current study using a controlled trial design, larger sample sizes and a more complete follow-up. Second, the screening of suitable participants might need to be improved. As motivation, readiness to change and psychological mindedness are likely to be crucial in self-help programs, ${ }^{14,18,19}$ valid screening instruments are necessary to increase the homogeneity of the sample and lower the dropout rate. Implementing booster sessions between the psychologist and the participant after completing a module may help sustain the increase in mental health.

It seems relatively easy to tailor Psyfit to the experiences of people with SCI, for example, by adding small phrases, for example, about a possible physical limitation, and replacing some exercises to ones suitable for wheelchair users. This would enable persons with SCI to feel their situation better acknowledged in the program. More flexibility would further enable participants to follow the program at their own desired pace. Finally, the compatibility of Psyfit with smartphones and tablets should be improved to keep pace with the current technological innovations. However, such adaptations are costly and might result in a decreased accessibility for financial reasons. Whether such an SCItailored and personalized program is more effective than a generic program remains to be investigated in future research.

\section{CONCLUSION}

The accessibility, affordability ( $€ 30$ per year) and the concise nature of Psyfit make this program a potential first step in treatment of depressive symptoms before considering more intensive and costly therapy in a Stepped-Care Model for a subgroup of people with SCI. Improvements concerning small content adaptations and program flexibility are recommended to increase its feasibility and satisfaction in persons with SCI.

\section{DATA ARCHIVING}

There were no data to deposit.

\section{CONFLICT OF INTEREST}

The affiliation of LB, Trimbos Institute, is the developer of Psyfit. Neither LB nor the institute itself derives financial outcome from this intervention.
1 Post MW, van Leeuwen CM. Psychosocial issues in spinal cord injury: a review. Spinal Cord 2012; 50: 382-389.

2 Sinnema H, Franx G, Spijker J, Ruiter M, Haastrecht van H, Verhaak $P$ et al. Delivering stepped care for depression general practice: results of a survey among general practitioners in the Netherlands. Eur J Gen Pract 2013; 19: 221-229.

3 Straten, van A, Hill J, Richards DA, Cuijpers P. Stepped care treatment delivery for depression: a systematic review and meta-analysis. Psychol Med 2014; 26: $1-16$

4 Fann JR, Crane DA, Graves DE, Kalpakjian CZ, Tate DG, Bombardier CH. Depression treatment preferences after acute traumatic spinal cord injury. Arch Phys Med Rehabil 2013; 94: 2389-2395.

5 Bolier L, Haverman M, Westerhof GJ, Riper H, Smit F, Bohlmeijer E. Positive psychology interventions: a meta-analysis of randomized controlled studies. BMC Public Health 2013; 13: 119.

6 Dorstyn D, Mathias J, Denson L. Applications of telecounselling in spinal cord injury rehabilitation: a systematic review with effect sizes. Clin Rehabil 2013; 27 1072-1083.

7 Migliorini C, Tonge B, Sinclair A. Developing and piloting ePACT: a flexible psychological treatment for depression in people living with chronic spinal cord injury. Behav Change 2011; 28: 45-54.

8 Bolier L, Haverman M, Kramer J, Westerhof GJ, Riper H, Walburg JA et al. An internetbased intervention to promote mental fitness for mildly depressed adults: randomized controlled trial. J Med Internet Res 2013; 15: e200.

9 van Leeuwen CM, van der Woude LH, Post MWM. Validity of the mental health subscale of the SF-36 in persons with spinal cord injury. Spinal Cord 2012; 50: 707-710.

10 Miller WC, Anton HA, Townson AF. Measurement properties of the CESD scale among individuals with spinal cord injury. Spinal Cord 2008; 46: 287-292.

11 Tennant R, Hiller L, Fishwick R, Platt S, Joseph S, Weich S et al. The WarwichEdinburgh Mental Well-being Scale (WEMWBS): development and UK validation. Health Qual Life Outcomes 2007; 5: 63.

12 Christensen H, Griffiths KM, Farrer L. Adherence in internet interventions for anxiety and depression. J Med Internet Res 2009; 11: e13.

13 Davis MJ, Addis ME. Predictors of attrition from behavioral medicine treatments. Ann Behav Med 1999; 21: 339-349.

14 Hall JA. Psychological-mindedness: a conceptual model. Am J Psychother 1992; 46 $131-140$.

15 Dorstyn DS, Mathias JL, Denson LA. Efficacy of cognitive behavioral therapy for the management of psychological outcomes following spinal cord injury: a meta-analysis. J Health Psychol 2011; 16: 374-391.

16 Dorstyn DS, Mathias JL, Denson LA. Psychosocial outcomes of telephone-based counselling for adults with an acquired physical disability: a meta-analysis. Rehabil Psychol 2011; 56: 1-14.

17 Kelders SM, Kok RN, Ossebaard HC, Van Gemert-Pijnen JE. Persuasive system design does matter: a systematic review of adherence to web-based interventions. J Med Internet Res 2012; 14: e152.

18 Tkachuk GA, Marshall JK, Mercado AC, McMurthy B, Stockdale-Winder F. Readiness to change predicts outcomes of functional rehabilitation following motor vehicle accident. J Occup Rehabil 2012; 22: 97-104.

19 Prochaska JO, Velicer WF, Rossi JS, Goldstein MG, Marcus BH, Rakowski W et al. Stages of change and decisional balance for 12 problem behaviors. Health Psychol 1994: 13: 39-46. 